\title{
Conformal Invariance and Conserved Quantities for Lagrange Equation of Thin Elastic Rod
}

\author{
Peng WAnG ${ }^{a, *}$, Hui-Rong Feng ${ }^{b}$ And Zhi-Mei Lou ${ }^{c}$ \\ ${ }^{a}$ School of Civil Engineering and Architecture, University of Jinan, Jinan, Shangdong, 250022, P.R. China \\ ${ }^{b}$ College of Transportation and Civil Engineering, Fujian Agriculture and Forestry University, Fuzhou, \\ Fujian, 350002, P.R. China \\ ${ }^{c}$ Department of Physics, Shaoxing University, Shaoxing, Zhejiang, 312000, P.R. China
}

(Received November 1, 2016)

\begin{abstract}
Basing on the analytical mechanics methods, the Lagrangian equations of thin elastic rod is constructed. The definition of conformal invariance for the Lagrange mechanics of elastic rod is given. The criterions that conformal invariance of elastic rod is the Lie symmetry are obtained based on the Lie point transformation group. The structure equation and conserved quantity deduced from conformal invariance of elastic rod are constructed. Take twist rod as an example to illustrate the application of the results got in this paper.
\end{abstract}

DOI: 10.12693/APhysPolA.131.283

PACS/topics: conformal invariance, Lie symmetry, conserved quantities, thin elastic rod, Lagrange equation

\section{Introduction}

The Lie group theory is a power tool to reduce the order of dynamic systems. An alternative method is to use the Lie point symmetry group to construct conserved quantities or first integrals. If a sufficient number of conserved quantities can be obtained by symmetries, exact solutions for dynamic systems can be achieved. The symmetry methods have been developed as modern tools to find conserved quantities of dynamic systems.

The research on symmetries and conserved quantities of mechanical systems possesses important theoretical and practical significance. The well known Noether symmetry has broad applications in mathematics, dynamics, and physics [1-7], it always can lead to conserved quantities. In fact, it is also named variational symmetry [3]. Besides the Noether symmetry, there are the Lie symmetry, the Mei symmetry, and so on [8-18]. Above symmetries are all basing on the Lie continuous transformation group. In 1997, Galiullin et al. [19] discussed the conformal invariance of Birkhoff system and deduced Noether conserved quantities from conformal invariance. Mei et al. [20] extended the conformal invariance to generalized Birkhoff equations and gave the Noether conserved quantities. The key question to the conformal invariance of dynamics is to find out the conformal factor. Considerable progress has made over past years in the application of conformal invariance to mechanical systems [21-26]. However, the application of conformal invariance to thin elastic rod has never been investigated.

The thin elastic rod model is adopted to describe the large deformation questions [27, 28], especially the DNA

*corresponding author; e-mail: sdpengwang@163.com supercoil $[29,30]$. It has been applied to model the configuration and the stability of super-helically constrained DNA [31-36]. Because of the special slender and superdeformation characteristics of the elastic rod model, its equation of motion is strongly nonlinear, which makes its solution difficult to be found. However, the symmetry under the Lie group transformation has its inherent applicability in classifying and reducing differential equations as well as in finding out conservation laws. So applying the symmetry to the elastic rod and finding out its conserved quantities via the symmetry analysis will be helpful for its research. Coleman et al. [37] introduced the first integrals and the variational principle for the rod dynamics. Maddocks et al. [38] gave vector integrals of motion for the rod dynamics and mentioned the corresponding symmetry transformation, but they did not give further discussion about symmetries. Fu et al. [39] studied the Noether symmetry of a superlong elastic rod in the Hamilton form. Jung et al. [40] studied a discrete method for special Cosserat elastic rod statics and gave the related Noether theorem. Xue et al. [41] studied the the conserved quantities in general theorems of elastic rod dynamics. In Refs. $[42,43]$ the authors studied the Mei symmetry and conserved quantities of Kirchhoff elastic rod statics and Noether theorem of Cosserat elastic rod dynamics. In Ref. [44] the authors studied the conformal invariance of Mei symmetry for thin elastic rod.

In the present paper, we study the conformal invariance of the Lagrange equation of thin elastic rod. We first describe the basic definitions of rod and give the Lagrange equation of elastic rod. Secondly, basing on the Lie point transformation group, we give the definition of conformal invariance of elastic rod and the criterion of necessary and sufficient condition that conformal invariance will be the Lie symmetry. Thirdly, we give the proposition that conformal invariance leads to conserved quantities. Finally, we take the twisted rod as example 
to illustrate the application of the results. The conserved quantities obtained in this paper can be helpful in the numerical simulation for DNA mechanics.

\section{Lagrange equation of elastic rod}

Consider an inextensible thin elastic rod of length $l$ with non-circular cross-section. Suppose the thin elastic rod is homogeneous and isotropic in mechanical properties along its length, and obeys linearly elastic constitutive relation. It is also assumed that no external force of torque is imposed on the rod except the two ends, i.e., the free elastic rod. Based on the Kirchhoff analogy the rod can be discretized into motion trajectory of the crosssection that moves along the center line of elastic rod in unit speed. The configuration of the cross-section can be described by the position of centroid which can be defined by the position vector, $\boldsymbol{R}(s)$, where $s$ is arc coordinate, and the attitude relative to centroid which can be defined by the Euler angles $(\psi(s), \theta(s), \varphi(s))$. In order to locate the configuration of the elastic rod, we establish the inertial coordinate system $O x y z$ with a fixed space origin $O$, and a principle coordinate system $O x^{*} y^{*} z^{*}$ with a centroid $O^{*}$ of cross-section. A space-fixed right-handed orthonormal frame $\left(\boldsymbol{e}_{x}, \boldsymbol{e}_{y}, \boldsymbol{e}_{z}\right)$ can be introduced for the inertial coordinate system. A body-fixed right-handed orthonormal frame $\left(\boldsymbol{d}_{1}, \boldsymbol{d}_{2}, \boldsymbol{d}_{3}\right)$ is chosen for principle coordinate system. The deformation geometric equation of center line of elastic rod satisfies

$$
\boldsymbol{R}^{\prime}=\boldsymbol{d}_{3}
$$

where the prime represents derivative with respect to arc coordinate $s$. Equation (1) denotes vector $\boldsymbol{d}_{3}$ in the direction of the local tangent of the neutral axis of elastic rod.

The change rate of attitude of cross-section relative to arc coordinate is called curvature-twisting vector, i.e. $\boldsymbol{\omega}_{s}$. Its components on the principle coordinate system are $\boldsymbol{\omega}_{s}=\omega_{1}(s) \boldsymbol{d}_{1}+\omega_{2}(s) \boldsymbol{d}_{2}+\omega_{3}(s) \boldsymbol{d}_{3}$ and has relation with the Euler angles as

$$
\begin{aligned}
& \omega_{1}=\psi^{\prime} \sin \theta \sin \varphi+\theta^{\prime} \cos \varphi, \\
& \omega_{2}=\psi^{\prime} \sin \theta \cos \varphi-\theta^{\prime} \sin \varphi, \\
& \omega_{3}=\psi^{\prime} \cos \theta+\varphi^{\prime} .
\end{aligned}
$$

The physical mean of curvature-twisting vector is the angular velocity of cross-section relative to inertial coordinate system when it moves along the center line of elastic rod in unit speed.

In fact, Eq. (1) is nonholonomic constraint, so the freedom degree of cross-section is three. Because it does not need external constraint force, so it is a pseudononholonomic constraint [45]. We choose the three Euler angles as independent variables to confirm the configuration of cross-section. The linear constitutive relation is taken in present paper. Expressing the Euler angles as generalized coordinates in analytical mechanics $q_{1}=\psi, q_{2}=\theta, q_{3}=\varphi$, and the $q_{j}^{\prime}$ denotes the derivative of the generalized coordinate to the arc coordinate and is named the generalized velocity, $q_{j}^{\prime \prime}$ denotes the second derivative of the generalized coordinate to arc coordinate and is named the generalized acceleration, neglecting the active force along the arc coordinate, we can get the Lagrange equation of elastic rod from the differential variational principle

$$
\frac{\mathrm{d}}{\mathrm{d} t} \frac{\partial \Gamma}{\partial q_{j}^{\prime}}-\frac{\partial \Gamma}{\partial q_{j}}+m_{q_{j}^{\prime}}^{G}=0 \quad(j=1,2,3),
$$

where $\Gamma$ is potential energy density function and is defined by:

$$
\Gamma=T-V,
$$

where $T$ is potential energy density function of strain and $V=-\boldsymbol{F} \cdot \boldsymbol{e}_{3}$. By the Kirchhoff dynamic analogy, $\Gamma$ can be regarded as Lagrangian function density of elastic rod, if $T$ can be regarded as kinetic energy and $V$ as potential energy, and $\boldsymbol{F}$ is internal force vector on the cross-section. $m_{q_{j}^{\prime}}^{G}$ is amount to active torque which may be seemed as non-potential generalized force. It should note that the arc coordinate variable is analogy to, by the Kirchhoff dynamic analogy, the time variable, so the movement of cross-section along the arc coordinate can be described by the Lagrange Eq. (4). From Eq. (4) we can have

$$
F_{j} \equiv A_{j k}(s, \boldsymbol{q}) q_{k}^{\prime \prime}+B_{j}\left(s, \boldsymbol{q}^{\prime}\right)=0 \quad(j, k=1,2,3),
$$

where $A_{j k}=\frac{\partial^{2} \Gamma}{\partial q_{j}^{\prime} \partial q_{k}^{\prime}}, B_{j}=\frac{\partial^{2} \Gamma}{\partial q_{j}^{\prime} \partial q_{k}} q_{k}^{\prime}+\frac{\partial^{2} \Gamma}{\partial q_{j}^{\prime} \partial t}-\frac{\partial \Gamma}{\partial q_{s}}$. Suppose the system is nonsingular, i.e., $D=\operatorname{det}\left(\frac{\partial^{2} \Gamma}{\partial q_{j}^{\prime} \partial q_{k}^{\prime}}\right)$, then all the generalized accelerations can be solved from Eq. (4) in the form

$$
q_{j}^{\prime \prime}=\frac{M_{j k}}{D}\left(\frac{\partial \Gamma}{\partial q_{k}}-\frac{\partial^{2} \Gamma}{\partial q_{k}^{\prime} \partial t}-\frac{\partial^{2} \Gamma}{\partial q_{k}^{\prime} \partial q_{j}^{\prime}} q_{j}^{\prime}+m_{q_{k}^{\prime}}^{G}\right),
$$

where $M_{j k}$ are co-factors of the matrix element $\frac{\partial^{2} \Gamma}{\partial q_{k}^{\prime} \partial q_{j}^{\prime}}$. Equation (6) can be further written as

$$
q_{j}^{\prime \prime}=\alpha_{j}\left(s, q_{j}, q_{j}^{\prime}\right), \quad j=1,2,3 .
$$

\section{Conformal invariance of the Lagrange equation of elastic rod}

In order to get the conform invariance of the Lagrange equation of elastic rod, we need to explore the transformation sets of independent or non-independent variables corresponding to Eqs. (3) or (5). Considering the symmetry of Eq. (5), we introduce a one-parameter infinitesimal Lie point transformation group in space $\left(s, q_{j}\right)$ :

$$
\begin{gathered}
s^{*}=s+\varepsilon \xi_{0}(s, \boldsymbol{q}), \quad q_{j}^{*}=q_{j}+\varepsilon \xi_{j}(s, \boldsymbol{q}), \\
j=1,2,3,
\end{gathered}
$$

where $\varepsilon$ is infinitesimal parameter, $\xi_{0}(s, \boldsymbol{q}), \xi_{j}(s, \boldsymbol{q})$ are infinitesimal transformation generators. It has infinitesimal generator vector

$$
X^{(0)}=\xi_{0} \frac{\partial}{\partial s}+\xi_{j} \frac{\partial}{\partial q_{j}},
$$

which is the operator for the infinitesimal generator of the one-parameter Lie group of transformations (9) in space $(s, \boldsymbol{q})$. The first prolongation of the infinitesimal generator vector is 


$$
X^{(1)}=X^{(0)}+\left(\xi_{s}^{\prime}-\xi_{0}^{\prime} q_{s}^{\prime}\right) \frac{\partial}{\partial q_{j}^{\prime}} .
$$

The second prolongation of the infinitesimal generator vector is

$$
X^{(2)}=X^{(1)}+\left(\xi_{j}^{\prime \prime}-2 q_{j}^{\prime \prime} \xi_{0}^{\prime}-q_{j}^{\prime} \xi_{0}^{\prime \prime}\right) \frac{\partial}{\partial q_{j}^{\prime \prime}},
$$

which defines a first or second extended one-parameter Lie group of transformation in space $(t, \boldsymbol{q}, \dot{\boldsymbol{q}})$ or space $(t, \boldsymbol{q}, \dot{\boldsymbol{q}}, \ddot{\boldsymbol{q}})$ by partial derivatives.

Definition 1. For elastic rod in the Lagrange form (3), if there exist a nonsingular matrix $\Pi_{s}^{l}$ satisfying

$$
X^{(2)}\left(F_{j}\right)=\Pi_{j}^{l}\left(F_{1}\right), \quad j, l=1,2,3,
$$

then the Lagrange equation of elastic rod (3) maintains conform invariance under the single parameter infinitesimal transformations (8).

Equation (12) is the determining equation of conform invariance of the Lagrange equation of elastic rod (3). $\Pi_{j}^{l}$ are the conformal factors of the system. If the infinitesimal generators $\xi_{0}(s, \boldsymbol{q}), \xi_{s}(s, \boldsymbol{q})$ satisfying the determining Eq. (12), then the transformation (8) is called conformal transformation.

Now the task is to how to find out the infinitesimal generators $\xi_{0}(s, \boldsymbol{q}), \xi_{s}(s, \boldsymbol{q})$ of conformal transformation and conformal factor $\Pi_{j}^{l}$. In order to get the conformal factor, one of the methods is that demands of the Lagrange equation of elastic rod have both conformal invariance and the Lie symmetry simultaneously under the infinitesimal transformations.

Proposition 1. For the Lagrange equation of elastic $\operatorname{rod}(3)$, if the generators $\xi_{0}(s, \boldsymbol{q}), \xi_{s}(s, \boldsymbol{q})$ of the infinitesimal transformations (8) satisfy the determining equation (12), and the exists a nonsingular matrix $\Lambda_{j}^{1}$ satisfying the following condition:

$$
X^{2}\left(F_{j}\right)-\left.X^{2}\left(F_{j}\right)\right|_{F_{j}=0}=\Lambda_{j}^{1} F_{j}, \quad j, l=1,2,3,
$$

then the necessary and sufficient condition that the conformal invariance would be the Lie symmetry of system (3) is

$$
\Pi_{j}^{k}=\Lambda_{j}^{k} .
$$

Proof: If the Lagrange equation of elastic rod (3) has the Lie symmetry, that is

$$
\left.X^{2}\left(F_{j}\right)\right|_{F_{j}=0}=0
$$

we can deduce the relation $\Pi_{j}^{k}=\Lambda_{j}^{k}$.

On the other hand, if the Lagrange equation of elastic rod (3) maintain conformal invariance, that is the determining Eq. (12) is verified, then we can get the system (3) is also the Lie symmetry from relation $\Pi_{j}^{k}=\Lambda_{j}^{k}$.

Calculating the difference of

$$
X^{2}\left(F_{j}\right)-\left.X^{2}\left(F_{j}\right)\right|_{F_{j}=0},
$$

we can get the conformal factor of conformal invariance of system (3). Expanding Eq. (16) and making use of the relation

$$
q_{k}^{\prime \prime}-\alpha_{k}=A^{k l} F_{1}, \quad k, l=1,2,3,
$$

we can get the difference

$$
X^{2}\left(F_{j}\right)-\left.X^{2}\left(F_{j}\right)\right|_{F_{j}=0}=B_{j}^{1} F_{1},
$$

$$
\begin{gathered}
B_{j}^{1}=A_{j k}\left(\frac{\partial \eta_{k}}{\partial q_{r}}-q_{r}^{\prime} \frac{\partial \xi_{0}}{\partial q_{r}}\right) A^{r l}-2 \delta_{j}^{1}\left(\frac{\partial \xi_{0}}{\partial s}+q_{r}^{\prime} \frac{\xi_{0}}{\partial q_{r}}\right) \\
\quad+X^{0}\left(A_{j k}\right) A_{k l}, \quad j, k, l=1,2,3 .
\end{gathered}
$$

We have the following corollaries to Proposition 1 .

Corollary 1. Under the infinitesimal group transformations (8), if the Lagrange equation of elastic rod (3) maintain both conformal invariance and Lie symmetry, then the conformal factor should be in the form of Eq. (19).

Corollary 2. If the Lagrange equation of elastic rod (3) can be expressed in the standard form

$$
F_{j} \equiv q_{j}^{\prime \prime}-\alpha_{j}\left(s, \boldsymbol{q}, \boldsymbol{q}^{\prime}\right)=0, \quad j=1,2,3,
$$

then the necessary and sufficient condition that the conformal invariance would be the Lie symmetry of system (3) is the conformal factor in the form

$$
B_{j}^{1}=\frac{\partial \eta_{j}}{\partial q_{1}}-2 \xi_{0}^{\prime}-q_{1}^{\prime} \frac{\partial \xi_{0}}{\partial q_{1}}, \quad j, l=1,2,3 .
$$

From the standard form (20), we can obtain $A_{s k}=\delta_{s k}$, $B_{j}(s, \boldsymbol{q}, \boldsymbol{q})=\alpha_{j}\left(s, \boldsymbol{q}, \boldsymbol{q}^{\prime}\right)$ in Eq. (5). So we can get Eq. (21) from Eq. (19).

\section{Conformal invariance and conserved quantities of the Lagrange equation of elastic rod}

As we know, there may exist conserved quantities corresponding to a symmetry. The conformal invariance can also lead to conserved quantities under certain limitation.

Proposition 2. If the infinitesimal generators $\left.\xi_{0}(s, \boldsymbol{q}), \eta_{j}(s, \boldsymbol{q})\right)$ satisfy the conformal factor (19), and there exists a gauge function $G_{N}\left(s, \boldsymbol{q}, \boldsymbol{q}^{\prime}\right)$ satisfying following structure equations, which are the sufficient and necessary conditions to deduce the Noether conserved quantities

$$
\Gamma \xi_{0}^{\prime}+X^{(1)}(\Gamma)+m_{q_{j}^{\prime}}^{G}\left(\eta_{j}-q_{j}^{\prime} \xi_{0}\right)+G_{N}^{\prime}=0,
$$

then the conformal invariance of the Lagrange equation of elastic rod (3) can lead to the conserved quantities in the following form:

$$
I_{N}=\Gamma \xi_{0}+\frac{\partial L}{\partial q_{j}^{\prime}}\left(\eta_{j}-q_{j}^{\prime} \xi_{0}\right)+G_{N}=\text { const. }
$$

The course of proof of proposition 2 can be referred in Ref. [1].

\section{Application of conformal invariance to thin elastic rod}

The potential energy density function in the generalized coordinate form for the circular cross-section of thin elastic rod is

$$
\Gamma=\frac{1}{2}\left(q_{1}^{\prime} \cos q_{2}+q_{3}\right)^{2}-F \cos q_{2} .
$$

The non-potential generalized forces are

$$
m_{q_{j}^{\prime}}^{G}=0 .
$$

The differential equations of motion of the system are

$$
\begin{aligned}
& F_{1}=q_{1}^{\prime \prime} \cos ^{2} q_{2}+q_{3}^{\prime \prime} \cos ^{2} q_{2}+2 q_{1}^{\prime} q_{2}^{\prime} \sin q_{2} \cos q_{2} \\
& -q_{2}^{\prime} q_{3}^{\prime} \sin q_{2}=0,
\end{aligned}
$$




$$
\begin{aligned}
& F_{2}=\left(q_{1}^{\prime}\right)^{2} \sin q_{2} \cos q_{2}+q_{1}^{\prime} q_{2}^{\prime} \sin q_{2}+F \sin q_{2}=0, \\
& F_{3}=q_{1}^{\prime} \cos q_{2}+q_{3}^{\prime}=0 .
\end{aligned}
$$

Taking the second prolongation of the infinitesimal generator vector to Eq. (26), we can get the determining equations of the Lie symmetry of system (26):

$$
\begin{aligned}
& \eta_{1}^{\prime \prime} \cos ^{2} q_{2}+\eta_{3}^{\prime \prime} \cos q_{2}-2 \eta_{1}^{\prime} q_{2}^{\prime} \sin q_{2} \cos q_{2} \\
& \quad-\eta_{1}^{\prime} \sin q_{2}\left(2 q_{1}^{\prime} \cos q_{2}+q_{3}^{\prime}\right)+\xi_{0}^{\prime \prime}\left(q_{1}^{\prime} \cos q_{2}-q_{3}^{\prime}\right) \cos q_{2} \\
& \quad-\xi_{0}\left(2 q_{1}^{\prime \prime}+2 q_{3}^{\prime \prime}+3 q_{1}^{\prime} q_{2}^{\prime} \sin q_{2}\right) \cos q_{2} \\
& \quad-2 \xi_{0}^{\prime} q_{2}^{\prime} q_{3}^{\prime} \sin q_{2}-\eta_{2}\left(2 q_{1}^{\prime \prime} \cos q_{2}-q_{3}^{\prime \prime}\right) \sin q_{2}, \\
& \quad-\eta_{2} q_{1}^{\prime} q_{2}^{\prime}\left(\cos ^{2} q_{2}-\sin ^{2} q_{2}\right)-\eta_{2} q_{2}^{\prime} q_{3}^{\prime} \cos q_{2}=0, \\
& \eta_{1}^{\prime}\left(2 q_{1}^{\prime} \cos q_{2}+q_{3}^{\prime}\right) \sin q_{2}+\eta_{3}^{\prime} q_{3}^{\prime} \sin q_{2} \\
& \quad-\xi_{0}^{\prime}\left(q_{1}^{\prime}\right)^{2} \sin q_{2} \cos q_{2}-2 \xi_{0}^{\prime} q_{1}^{\prime} q_{3}^{\prime} \sin q_{2} \\
& \quad+\eta_{2}\left(q_{1}^{\prime}\right)^{2}\left(\cos ^{2} q_{2}-\sin ^{2} q_{2}\right) \\
& \quad+\eta_{2}\left(q_{1}^{\prime} q_{3}^{\prime}+F\right) \cos q_{2}=0, \\
& \eta_{1}^{\prime} \cos q_{2}+\eta_{3}^{\prime}-\xi_{0}^{\prime}\left(q_{1}^{\prime} \cos q_{2}-q_{3}^{\prime}\right) \\
& \quad-\eta_{2} q_{1}^{\prime} \sin q_{2}=0,
\end{aligned}
$$

when the infinitesimal generators taking the value of

$$
\xi_{0}=1, \eta_{1}=-q_{2}, \eta_{2}=0, \eta_{3}=\sin q_{2},
$$

Eqs. (27) are verified, that is to say, generators (28) are the Lie symmetrical. We can get the conformal factor from (19) or (21) as

$$
\Pi_{j}^{1}=\left(\begin{array}{ccc}
0 & -1 & 0 \\
0 & 0 & 0 \\
0 & \cos q_{2} & 0
\end{array}\right),
$$

so the determining equation of conformal invariance of system (26) is

$$
X^{2}\left(\begin{array}{l}
F_{1} \\
F_{2}
\end{array}\right)=\left(\begin{array}{ccc}
0 & -1 & 0 \\
0 & 0 & 0 \\
0 & \cos q_{2} & 0
\end{array}\right)\left(\begin{array}{l}
F_{1} \\
F_{2}
\end{array}\right) .
$$

When the infinitesimal generators take the value of

$$
\xi_{0}=1, \quad \eta_{1}=1, \quad \eta_{2}=0, \quad \eta_{3}=1,
$$

Eq. (27) is satisfied. However, because the conformal factor corresponding to generators (31) is null matrix, so the generators (31) do not satisfy conformal invariance. We can conclude again that the generators of the Lie symmetry to be conformal invariance have to satisfy the condition (13).

Taking the generators (28) into structure equation (22), we can work out the gauge function

$$
G_{N}=\text { const, }
$$

so we can get the conserved quantities corresponding to conformal invariance of Lagrange equation of elastic rod as

$$
\begin{aligned}
& I_{N}=\frac{1}{2} C\left(q_{1}^{\prime} \cos q_{2}+q_{3}^{\prime}\right)^{2} \\
& \quad-C\left(q_{2}+q_{1}^{\prime}\right)\left(q_{1}^{\prime} \cos ^{2} q_{2}+q_{3}^{\prime} \cos q_{2}\right) \\
& \quad+C\left(q_{1}^{\prime} \cos q_{2}+q_{3}^{\prime}\right)\left(\sin q_{2}-q_{3}^{\prime}\right) \\
& \quad-F \cos q_{2}+G_{N}=\text { const. }
\end{aligned}
$$

For infinitesimal generators (31), we can get conserved quantities deduced from the Lie symmetry of system

$$
I_{N}=\frac{1}{2} C\left(q_{1}^{\prime} \cos q_{2}+q_{3}^{\prime}\right)^{2}
$$

$$
\begin{aligned}
& -C\left(1+q_{1}^{\prime}\right)\left(q_{1}^{\prime} \cos ^{2} q_{2}+q_{3}^{\prime} \cos q_{2}\right) \\
& +C\left(q_{1}^{\prime} \cos q_{2}+q_{3}^{\prime}\right)\left(1-q_{3}^{\prime}\right)-F \cos q_{2}=\text { const. }
\end{aligned}
$$

They are two types of new conserved quantities for superthin elastic rod which are never got in the past.

\section{Conclusion}

The superthin elastic rod models have broad applications in engineering and biology. The differential equations of motion of superthin elastic rod are strongly nonlinear for its large deformation. This paper utilizes the analytical mechanics methods to construct the Lagrange equations of rod, and applies the conformal invariance to thin elastic rod Lagrange mechanics, and gives the corresponding conserved quantities to this symmetry.

The definition of conformal invariance of elastic rod is given. In order to find the conformal factor, the proposition 1 and two corollaries are proposed. They are also the necessary and sufficient condition that the Lie symmetry would be conformal invariance of the system. The proposition 2 gives conserved quantities of the conformal invariance of loaded rod. We take the rod with twist rate as an example to illustrate the application of the results got in this paper. The case of general elastic rod needs further study.

\section{Acknowledgments}

This work is partly supported by National Natural Science Foundation of China grant Nos. 11262019, 11472177, and the Natural Science Foundation of Fujian Province, grant No. 2016J01664.

\section{References}

[1] F. Mei, Symmetries and Conserved Quantities of Constrained Mechanical Systems, Beijing Institute of Technology Press, Beijing 2004 (in Chinese).

[2] R. de Ritis, G. Marmo, G. Platania, C. Rubano, P. Scudellaro, C. Stornaiolo, Phys. Rev. D 42, 1091 (1990).

[3] V. Dorodnitsyn, Appl. Num. Math. 39, 307 (2001).

[4] D. Levi, P. Winternitz, J. Phys. A Math. Gen. 39, 1 (2006).

[5] P. Wang, Nonlin. Dyn. 68, 53 (2012).

[6] P. Wang, H.J. Zhu, Acta Phys. Pol. A 119, 298 (2011).

[7] Z.X. Long, Y. Zhang, Acta Mech. 225, 77 (2013).

[8] M. Lutzky, Int. J. Non-Lin. Mech. 34, 387 (1999).

[9] F.X. Mei, Acta Mech. 141, 135 (2000).

[10] F.X. Mei, Chin. Phys. 10, 177 (2001).

[11] J.H. Fang, Chin. Phys. B 19, 040301 (2010).

[12] L.Q. Jia, X.X. Wang, M.L. Zhang, Y.L. Han, Nonlin. Dyn. 69, 1807 (2012).

[13] S.K. Luo, Z.J. Li, W. Peng, L. Li, Acta Mech. 224 71 (2013).

[14] S.K. Luo, Y.L. Xu, Int. J. Theor. Phys. 54, 572 (2015). 
[15] S.K. Luo, Z.J. Li, L. Li, Acta Mech. 223, 2621 (2012).

[16] F.X. Mei, H.B. Wu, Y.F. Zhang, Int. J. Dyn. Control. 2, 285 (2014).

[17] Z.M. Lou, Acta Phys. Sin. 62, 220201 (2013) (in Chinese).

[18] C.J. Song, Y. Zhang, Int. J. Theor. Phys. 54, 2481 (2015).

[19] A.S. Galiullin, G.G. Gafarov, R.P. Malaishka A.M. Khwan, Analytical Dynamics of Helmholtz, Birkhoff and Nambu Systems, UFN, Moscow 1997.

[20] F.X. Mei, J.F. Xie, T.Q. Gang, Acta Mech. Sin. 24, $583(2008)$.

[21] C. Liu, F.X. Mei, Y.X. Guo, Acta Phys. Sin. 57, 6704 (2008) (in Chinese).

[22] X.W. Chen, Y.M. Li, Y.H. Zhao, Chin. Phys. B 18 3139 (2009)

[23] Y.P. Luo, J.L. Fu, Chin. Phys. B 19, 090303 (2010).

[24] J.L. Cai, Int. J. Theor. Phys. 49, 201 (2010).

[25] J.L. Cai, Nonlin. Dyn. 69, 487 (2012).

[26] Y.Y. Zhang, F. Zhang, Y.L. Han, L.Q. Jia, Nonlin. Dyn. 77, 521 (2014).

[27] E.E. Zajac, Trans. ASME J. Appl. Mech. 29, 136 (1962).

[28] G.H.M. Van der Heijden, Proc. R. Soc. Lond. A 457 , 695 (2001).

[29] C.J. Benham, Proc. Natl. Acad. Sci. USA 74, 2937 (1977).

[30] Y.Z. Liu, Nonlinear Mechanics of Thin Elastic RodTheoretical Basis of Mechanical Model of DNA, Tsinghua Press and Springer, Beijing 2006 (in Chinese).
[31] Y.M. Shi, J.E. Hearst, J. Chem. Phys. 101, 5186 (1994).

[32] R.S. Manning, K.A. Rogers, J.H. Maddocks, Proc. R. Soc. Lond. A 454, 3047 (1998).

[33] A. Goriely, M. Tabor, Phys. Rev. Lett. 77, 3537 (1996).

[34] I. Tobias, D. Swigon, B.D. Coleman, Phys. Rev. E 61, 747 (2000).

[35] Y. Xue, Y.Z. Liu, Acta Phys. Sin. 5, 6737 (2009) (in Chinese).

[36] Y.Z. Liu, Y. Xue, Appl. Math. Mech. Engl. Ed. 32 , 603 (2011).

[37] B.D. Coleman, E.H. Dill, D. Swigon, Archiv. Ration. Mechan. Anal. 129, 147 (1995).

[38] J.H. Maddocks, D.J. Dichmann, J. Elastic. 34, 83 (1994).

[39] J.L. Fu, W.J. Zhao, Y.Q. Weng, Chin. Phys. 17, 2361 (2008).

[40] P. Jung, S. Leyendecker, J. Linn, M. Ortiz, Int. J. Num. Meth. Eng. 85, 31 (2011).

[41] Y. Xue, P. Wang, Acta Phys. Sin. 60, 114501 (2011) (in Chinese).

[42] P. Wang, Y. Xue, Y.L. Liu, Chin. Phys. B 21, 070203 (2012).

[43] P. Wang, Y. Xue, Y.L. Liu, Chin. Phys. B 22, 104503 (2013).

[44] P. Wang, Y. Xue, Nonlin. Dyn. 83, 1815 (2016).

[45] Y. Xue, Y.Z. Liu, L.Q. Chen, Chin. J. Theor. Appl. Mech. 37, 485 (2005) (in Chinese). 